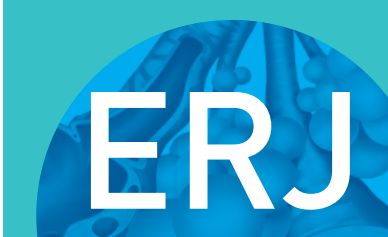

open research

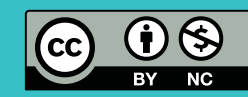

\section{Sleep breathing disorders: have we reached the tipping point?}

\author{
Monique C. Suarez-Giron ${ }^{1}$, Valentina Isetta ${ }^{2}$, Juan F. Masa ${ }^{3,4}$, Carlos Egea ${ }^{4,5}$, \\ Renata L. Riha6 ${ }^{6}$, Maria R. Bonsignore ${ }^{7,8}$ and Josep M. Montserrat ${ }^{1,4}$ \\ ${ }^{1}$ Sleep Unit, Pulmonary Service, Hospital Clinic, Barcelona, Spain. ${ }^{2}$ Unit of Biophysics and Bioengineering, \\ Faculty of Medicine, University of Barcelona, Barcelona, Spain. ${ }^{3}$ Pulmonary Service, Hospital San Pedro de \\ Alcantara, Cáceres, Spain. ${ }^{4}$ Centro de Investigación Biomédica en Red de Enfermedades Respiratorias \\ (CIBERES), Madrid, Spain. ${ }^{5}$ Sleep Unit, Respiratory Dept, Alava University Hospital IRB, Vitoria, Spain. ${ }^{6}$ Dept of \\ Sleep Medicine, Royal Infirmary Edinburgh, Edinburgh, UK. ${ }^{7}$ Biomedical Department of Internal and \\ Specialistic Medicine (DiBiMIS), University of Palermo, Palermo, Italy. ${ }^{8}$ Institute of Biomedicine and Molecular \\ Immunology (IBIM), National Research Council (CNR), Palermo, Italy.
}

Correspondence: Josep M. Montserrat, Sleep Unit, Pulmonary Service, Hospital Clinic, Villarroel 170, 08036, Barcelona, Spain. E-mail: jmmontserratQub.edu

\section{@ERSpublications}

Sleep medicine: a new approach http://ow.ly/qzVh30iVkWL

Cite this article as: Suarez-Giron MC, Isetta V, Masa JF et al. Sleep breathing disorders: have we reached the tipping point? ERJ Open Res 2018; 4: 00172-2017 [https://doi.org/10.1183/ 23120541.00172-2017].

In recent decades, the study of sleep breathing disorders has accelerated and increased our overall knowledge of sleep disorders in general. This now represents a real challenge to the health system due to the high prevalence, morbidity and mortality with concomitant social and economic repercussions [1].

Over the last few years, with sleep apnoea having greater diffusion on social media and in professional areas, the demands of sleep care have increased exponentially; however, health system strategies have not provided an adequate response. This has resulted in the saturation of sleep units with long patient waiting times, especially at a tertiary care level. This is far removed from the usual logical consideration that in the case of a common disease, all levels of care must be involved, from primary care centres, nonreferring hospitals, specialised nursing units and administration, to homecare providers, in order to deal with patients in an appropriate and personalised way. However, this basic concept of medical care collides with what is, at present, an embarrassing reality. Global sleep disorders are not dealt with in any detail at universities and little attention is paid to them during residencies or medical training periods. In fact, a percentage of respiratory specialists have no knowledge of sleep apnoea or other associated sleep disorders [2].

As a consequence, health professionals at various medical care levels find it difficult to carry out diagnosis, treatment and follow-up or even appropriate referral. In addition, it is well known that symptoms are the most important indication for treatment but there are other deleterious effects that must be taken into account. For instance, at present, sleep apnoea syndrome (SAHS) is "stagnant" in cardiovascular patients but if we consider it as a general entity, its harmful effects may occur in other settings such as metabolic and neurocognitive disorders, renal pathology, or even cancer [3-6]. Moreover, sleep breathing disorders are not restricted to SAHS, which in noncomplex situations, can be controlled by primary care medicine, but also include breathing disorders that need noninvasive mechanical ventilation, which is likely to establish itself as a key element in the near future, thanks in part to advances in technology. However, technology itself can also be harmful if not properly used or understood, a phenomenon known as the technological labyrinth syndrome. Technology should be simple, easy to use, reliable and above all, transparent; it should not be a black box. The better it is understood, the better it will be used. Other sleep disorders should be borne in mind, such as insomnia, chronobiology, paediatric sleep disorders and sleep

Received: Dec 272017 | Accepted after revision: Feb 112018

Copyright $\odot$ ERS 2018. This article is open access and distributed under the terms of the Creative Commons Attribution Non-Commercial Licence 4.0. 
health, which make up an important quintet both due to their symptoms and due to the risk they represent for other diseases [7,8]. At present, the paediatric population needs more attention, as do women with SAHS; two areas that have received minimum attention to date.

All the problems listed above are clamouring for a sea change in the way we focus on our constantly changing reality and new challenges [9]. Networking and telemedicine techniques seem to be a good option, possibly generating a cost-effective personalised form of care provision. However, this has not been universally adopted and the care provided in sleep medicine in certain countries is probably outdated [10]. As our knowledge changes in the light of new technologies, methods and research [11], so does the stability of the status quo. Another aspect to bear in mind is the fact that sleep medicine is often led by service companies, insurers or even government bodies, thus disproportionately driving practice [11, 12]. Medical care must not be a monetary concern but rather a health concern, and in relation to sleep disorders, should focus on the effect that these disorders have on the rest of the organism in health and disease. Sleep is probably even more important than exercise; for example, 2 days without proper sleep can have a more deleterious effect on health than not performing physical exercise or following an adequate diet (two of the classic elements of health).

Should a new approach to sleep medicine be based mainly on evolution, on disruption or on both? Evolution can be slow but fast disruption may be dangerous. To answer this question, a number of considerations should be made to realise that the present needs with respect to sleep in a broad spectrum are initially sufficient to adjust the necessary change, and in the context of illness and health. In order to introduce sleep into the concept of general health, it will be essential to liaise with the authorities, with all health professionals, with the population at large and with others who work to improve health through sleep. The broadcasting of regulations is also a key element.

Regarding sleep as a clinical entity, several fundamental aspects of our understanding and practice require modification. 1) Health professionals must start to adapt to new scenarios; care for sleep as an element of health in general terms must be provided by all professionals in collaboration with companies, the health authorities and universities. 2) Patient assessment must improve in order to personalise management and attempt to be mainly outpatient based. 3) Most current technologies for assessing sleep (polysomnography) should improve (new sensors, simple devices and contactless equipment) and most of them should be able to record data over several days. 4) Collaboration across different professions and medical specialties, epidemiologists, basic researchers, and biomedical engineers must become the defining premise that will allow us to learn and understand what patients and professionals need to carry our transformative action. All levels of care must be involved and technology must become simple to use, reliable and transparent regarding its algorithms and programmes.

In view of the speed of current global changes, a good example of evolution could be the application of lean methodology: continuous, linear improvement in the short term, which in fact represents a disruptive aspect. In this regard, it is essential to develop, prioritise the reasons for our projects, and think of sleep as a combination of entities that may affect other biological systems in both disease and health. These two viewpoints have led to different opinions or positions in different societies [13-15]. Perhaps it is not only a problem of urgency but above all, one of mentality and adaptation. At present, the quintet of sleep breathing disorders, insomnia, chronobiology in a broad sense, paediatrics and sleep health are the first imperative challenges to address. The different levels of care, collaboration with patients and the proper use of new technology are key elements that are essential to approach to sleep disturbances.

These points of view are those of the authors and not a position statement of the European Respiratory Society.

Conflict of interest: None declared.

\section{References}

1 Hafner M, Stepanek M, Taylor J, et al. Why sleep matters - the economic costs of insufficient sleep. A cross-country comparative analysis Published by the RAND Corporation, Santa Monica, CA, USA, and Cambridge, UK. https://www.rand.org/randeurope/research/projects/the-value-of-the-sleep-economy.html

2 Hayes SM, Murray S, Castriotta RJ, et al. (Mis)perceptions and interactions of sleep specialists and generalists: obstacles to referrals to sleep specialists and the multidisciplinary team management of sleep disorders. J Clin Sleep Med 2012; 8: 633-642.

3 Ahmed SB. Can treatment of obstructive sleep apnea with CPAP still improve kidney outcomes? Am J Respir Crit Care Med 2017; 196: 1370-1371.

4 Tahrani AA. Obstructive sleep apnoea in diabetes: does it matter? Diab Vas Dis Res 2017; 14: 454-462.

5 Bucks RS, Olaithe M, Rosenzweig I, et al. Reviewing the relationship between OSA and cognition: where do we go from here? Respirology 2017; 22: 1253-1261. 
6 Almendros I, Montserrat JM, Ramírez J, et al. Intermittent hypoxia enhances cancer progression in a mouse model of sleep apnoea. Eur Respir J 2012; 39: 215-217.

7 Bandla H, D'Andrea LA. Natural history and management of pediatric obstructive sleep apnea emerging concepts. Sleep 2015; 38: 11-12.

8 Mukherjee S, Patel SR, Kales SN, et al. An official American Thoracic Society statement: the importance of healthy sleep. Recommendations and future priorities. Am J Respir Crit Care Med 2015; 191: 1450-1458.

9 Doarn CR, Merrell RC. Are you a disruptor and is anyone paying attention? Telemed J E Health 2015; 21: 319-320.

10 Shelgikar AV, Durmer JS, Joynt KE, et al. Multidisciplinary sleep centers: strategies to improve care of sleep disorders patients. J Clin Sleep Med 2014; 10: 693-697.

11 Thomas RJ, Bianchi MT. Changing the direction of sleep medicine: business can boom, but it is not as usual. J Clin Sleep Med 2013; 9: 977-979.

12 Thomas RJ, Guilleminault C, Ayappa I, et al. Scoring respiratory events in sleep medicine: who is the driver biology or medical insurance? J Clin Sleep Med 2014; 10: 1245-1247.

13 Watson NF, Rosen IM, Chervin RD. The past is prologue: the future of sleep medicine. J Clin Sleep Med 2017; 13: $127-135$.

14 Lim DC, Pack AI. Obstructive sleep apnea: update and future. Annu Rev Med 2017; 68: 99-112.

15 Pack AI. What can sleep medicine do? J Clin Sleep Med 2013; 9: 629. 